\title{
Lack of Baseline Intracranial Aneurysm Wall Enhancement Predicts Future Stability: A Systematic Review and Meta-Analysis of Longitudinal Studies
}

\author{
(D) A.S. Larson, (D) V.T. Lehman, (D) G. Lanzino, and (D). Brinjikji
}

\begin{abstract}
BACKGROUND: The utility of vessel wall MR imaging in identifying unstable intracranial aneurysms has been suggested but remains controversial.

PURPOSE: Our aim was to provide further insight into the potential relationship between aneurysm wall enhancement on initial vessel wall imaging and aneurysm instability at follow-up.
\end{abstract}

DATA SOURCES: Our sources were PubMed, Scopus, the Web of Science, and the Cochrane Central Register of Controlled Trials.

STUDY SELECTION: We searched for English language studies that reported the presence of vessel wall enhancement of unruptured intracranial aneurysms on baseline vessel wall imaging studies with longitudinal follow-up of aneurysm status.

DATA ANALYSIS: Aneurysms were grouped into "stable" and "unstable" groups at follow-up on the basis of growth, symptomatic manifestation, or rupture. The association of each group with aneurysm wall enhancement on initial vessel wall imaging was determined.

DATA SYNTHESIS: Three studies constituting 407 aneurysms were included. Aneurysms with wall enhancement were at higher risk of being unstable at follow-up (risk ratio $=3.6 ; 95 \%$ confidence interval, 1.7-7.5). The sensitivity of aneurysm wall enhancement on vessel wall imaging was $74.3 \%(95 \% \mathrm{Cl}, 56.7 \%-87.5 \%)$, specificity was $58.3 \%$ (95\% Cl, $53.1 \%-63.4 \%)$, positive predictive value was $14.4 \%$ (95\% Cl, $11.8 \%-17.4 \%)$, negative predictive value was $96.0 \%(95 \% \mathrm{Cl}, 93.2 \%-97.7 \%)$, and the overall accuracy of the test was $59.7 \%$ (95\% Cl, 54.8\%-64.5\%).

LIMITATIONS: Only 3 studies were identified for inclusion in this analysis. More longitudinal studies of vessel wall imaging and aneurysm progression are needed.

CONCLUSIONS: The lack of wall enhancement may be a predictor of aneurysm stability. The utility of vessel wall imaging in detecting unstable aneurysms requires more data.

ABBREVIATIONS: AWE = aneurysm wall enhancement; IA = intracranial aneurysm; VWI = vessel wall imaging

ntracranial saccular aneurysms (IAs) are common vascular lesions with an estimated prevalence of up to $3 \% .^{1}$ In patients with unruptured IAs, it is challenging to know which IAs should be managed conservatively and which are at higher risk of rupture, thereby warranting intervention. Several patient-based factors and imaging features including

Received April 6, 2020; accepted after revision June 5.

From the Departments of Radiology (A.S.L., V.T.L., G.L., W.B.) and Neurosurgery (A.S.L., G.L., W.B.), Mayo Clinic, Rochester, Minnesota.

Please address correspondence to Waleed Brinjikji, BS, Mayo Clinic, 200 1st St SW, Rochester MN, 55905; e-mail: Brinjikji.Waleed@mayo.edu; @TonyLarsonBS; @WBrinjikji

Indicates article with supplemental on-line appendix and tables.

http://dx.doi.org/10.3174/ajnr.A6690 size, aspect ratio, and irregular morphology have been shown to be risk factors for IA rupture. ${ }^{2-6}$ Nevertheless, risk stratification of patients with unruptured IA remains inadequate; thus, the need for additional tools remains high in order to maximize our ability to appropriately select patients for intervention.

Inflammation within the vessel wall of the aneurysm is associated with IA instability and rupture. ${ }^{7,8}$ With advances in imaging, aneurysm wall enhancement (AWE) on vessel wall imaging (VWI) is purported to be a marker of aneurysmal wall inflammation and, therefore, a potential marker for aneurysm growth and rupture. ${ }^{9-11}$ Initial studies suggest that AWE is a marker of instability. However, authors have questioned the strength of the current evidence on several grounds. For example, there is some 


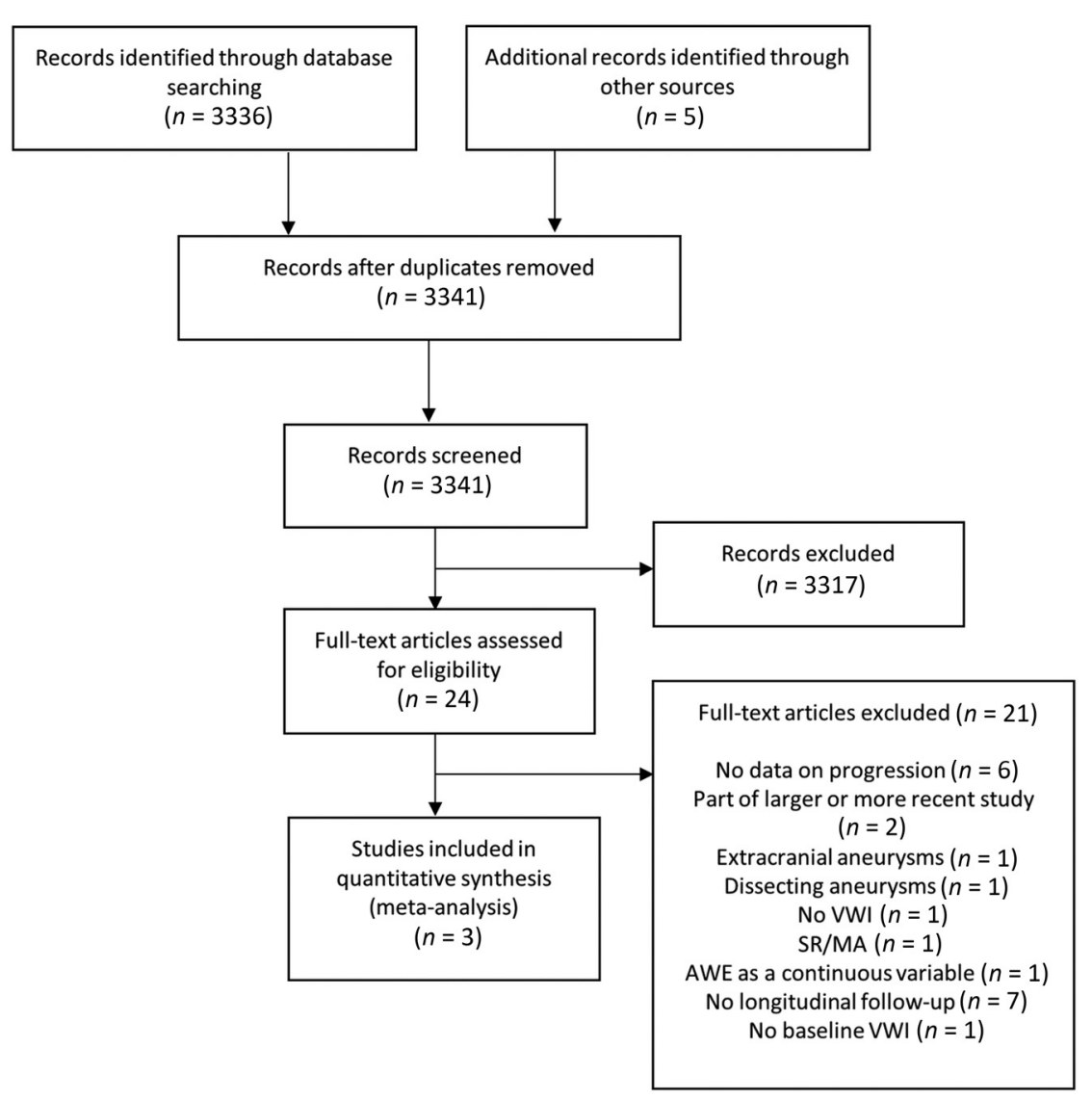

FIGURE. Literature review search strategy. SR/MA indicates systematic review/meta-analysis.

\section{MATERIALS AND METHODS Search Strategy and Selection Criteria}

Both the systematic review and metaanalysis were performed according to the Preferred Reporting Items for Systematic Reviews and Meta-Analyses guidelines. ${ }^{20} \mathrm{~A}$ comprehensive search of several data bases from inception to March 10, 2020, limited to the English language and excluding animal studies, was conducted. The data bases included Ovid MEDLINE and Epub Ahead of Print, In-Process \& Other Non-Indexed Citations, Ovid MEDLINE Daily, Ovid EMBASE, Ovid Cochrane Central Register of Controlled Trials, Ovid Cochrane Data Base of Systematic Reviews, and Scopus. The search strategy was designed and conducted by an experienced librarian with input from the principal investigator of the study. Controlled vocabulary supplemented with keywords was used to search for studies describing vessel wall imaging and intracranial aneurysms. The actual strategy listing all search terms used and how they are combined is available in the On-line Appendix.

Abstracts were reviewed for relevance, which included mention of in-

evidence that AWE can be caused by factors other than inflammation, such as slow blood flow near the aneurysm wall. ${ }^{12}$ Additionally, the literature demonstrates grouping of types of unstable aneurysms in analyses, lack of robust histopathologic correlation, variability of methods, a possible association with increasing size, and, most important, limited long-term follow-up. ${ }^{13,14}$ Until such prospective standardized long-term follow-up can be completed on a large number of patients, continued, periodic analysis of all available publications is important to characterize our best understanding of the current literature.

A prior meta-analysis demonstrated a robust relationship between AWE and aneurysm instability, ${ }^{11}$ but only a single included study had data on longitudinal follow-up. ${ }^{15}$ Since then, additional studies reporting longitudinal follow-up after initial VWI have been published; therefore, a separate analysis of these studies may shed more light on the clinical utility of VWI in detecting aneurysms at risk for progression. ${ }^{16-19}$ Inclusion of ruptured aneurysms in unstable aneurysm subgroups likely plays a confounding role; therefore, ruptured aneurysms should be analyzed independently or not included in such analyses. With these ideas in mind, we performed a systematic review and meta-analysis of longitudinal studies to provide further insight into the controversial relationship between AWE on VWI and aneurysm instability. tracranial aneurysms and vessel wall imaging. Abstracts without mention of both of these variables were excluded, and the full text was not reviewed. A study was considered eligible for this meta-analysis if it fulfilled the predefined inclusion criteria: Prospective and retrospective observational cohort analysis that compared the rates of AWE on initial VWI with longitudinal follow-up of aneurysm status. Our search strategy can be found in Fig 1.

\section{Data Extraction and Aneurysm Classification}

One reviewer (A.S.L.) independently extracted the relevant data from the eligible studies. Data extraction was performed for the following predefined variables: first author, year of publication, total number of patients, number of aneurysms demonstrating complete or partial AWE on initial vessel wall MR imaging, follow-up period, mean aneurysm size, and the number of stable and unstable intracranial saccular aneurysms as defined below. Also, data regarding the imaging sequences and field strength, interobserver agreement, and definition of wall enhancement were extracted. The primary end point of this meta-analysis was to compare complete or partial AWE as seen on initial vessel wall MR imaging studies between aneurysms that were stable or unstable at follow-up. In this regard, AWE was determined on a yes or no basis regardless of the degree or spatial pattern of enhancement. The definition of unstable saccular aneurysms included symptomatic, growing aneurysms on serial imaging or 
those that ruptured at follow-up. Symptomatic aneurysms included intracranial aneurysms causing cranial nerve palsy or thunderclap headache without subarachnoid hemorrhage and after exclusion of other differential diagnoses. ${ }^{16}$ The risk of bias assessment was performed with the Robins-I tool for nonrandomized studies (https:// www.riskofbias.info/welcome/home). ${ }^{21}$

\section{Statistical Synthesis and Analysis}

Risk ratios with the corresponding 95\% confidence intervals were used to report the outcomes. A $P$ value $<.05$ was considered significant. We also calculated sensitivity, specificity, positive predictive value, negative predictive value, and accuracy of AWE in identifying unstable aneurysms at follow-up. STATA, Version 14.1 (StataCorp) was used as statistical software.

\section{RESULTS}

\section{Search Results}

The abstracts and titles from a total of 3341 records were reviewed for relevance. From this number, 3317 were excluded. Twenty-four full-text articles were reviewed, 21 of which were excluded for various reasons: Six were excluded because whether or not the aneurysm analyzed with VWI was growing or symptomatic was not mentioned in the text, 2 were excluded because they belonged to a larger or more recent study from the same group, 1 study was of extracranial aneurysms, 1 study was of dissecting aneurysms, 1 did not contain VWI studies of aneurysms, and 1 was a systematic review/meta-analysis. One study reported AWE as a continuous variable, which precluded our ability to consider AWE as a binary variable (with or without enhancement), and this study was therefore excluded. ${ }^{22}$ Seven studies did not have longitudinal follow-up, and 1 did not have baseline VWI. Three studies satisfied the predetermined search criteria and were included in this meta-analysis as shown in the Preferred Reporting Items for Systematic Review and Meta-Analysis flow diagram (Figure). ${ }^{16,18,19}$

\section{Characteristics of Studies and Aneurysms}

All included studies in this meta-analysis were observational cohort analyses with longitudinal follow-up. In total, 407 saccular aneurysms with baseline VWI performed with follow-up analysis of aneurysm status (growth, symptom manifestation, or rupture) were included in this study. Detailed study characteristics are presented in On-line Table 1. Imaging characteristics are presented in On-line Table 2. All studies were determined to have a moderate risk of bias primarily attributable to their retrospective design and inherent risk of bias (On-line Table 3).

\section{Vessel Wall Enhancement of Unstable-versus-Stable Intracranial Saccular Aneurysms}

Aneurysms with AWE on initial VWI had a statistically significant higher risk of being unstable at follow-up (risk ratio $=3.6$; 95\% CI, 1.7-7.5) (Table 1). The sensitivity of AWE on VWI was $74.3 \%(95 \%$ CI, $56.7 \%-87.5 \%$ ), specificity was $58.3 \%$ (95\% CI, 53.1\%-63.4\%), positive predictive value was $14.4 \%$ ( $95 \% \mathrm{CI}, 11.8 \%-17.4 \%)$, negative predictive value was $96.0 \%$ (95\% CI, 93.2\%-97.7\%), and the overall accuracy of the test was $59.7 \%$ (95\% CI, 54.8\%-64.5\%) (Table 2).
Table 1: Association of stable and unstable aneurysms with wall enhancement on initial VWI studies

\begin{tabular}{lccc}
\hline & Unstable & Stable & Risk Ratio (95\% CI) \\
\hline Wall enhancement & 26 & 155 & $3.6(1.7-7.5)$ \\
No wall enhancement & 9 & 217 & \\
\hline
\end{tabular}

Table 2: Diagnostic statistics of aneurysm wall enhancement seen on VWI studies

\begin{tabular}{lc}
\hline \multicolumn{1}{c}{ Statistic } & Value (95\% Cl) \\
\hline Sensitivity & $74.29 \%(56.7 \%-87.5 \%)$ \\
Specificity & $58.3 \%(53.1 \%-63.4 \%)$ \\
Positive predictive value & $14.4 \%(11.8 \%-17.4 \%)$ \\
Negative predictive value & $96.0 \%(93.2 \%-97.7 \%)$ \\
Accuracy & $59.7 \%(54.8 \%-64.5 \%)$ \\
\hline
\end{tabular}

\section{DISCUSSION}

Our meta-analysis of 407 saccular aneurysms that underwent baseline VWI with longitudinal follow-up demonstrated 2 primary findings: First, the high negative predictive value (96.0\%) found here indicates that AWE on VWI may be a useful method of broadly screening aneurysms for stability in that those without AWE are exceptionally unlikely to progress. Second, the low positive predictive value of $14.4 \%$ suggests that a considerable number of IAs that demonstrate AWE may actually be stable.

We found a high negative predictive value of AWE for aneurysm instability. Other cross-sectional and longitudinal studies and a prior meta-analysis including predominantly cross-sectional data have found similar results. Edjlali et $\mathrm{al}^{16}$ reported on 263 patients with a total of 333 aneurysms and found the negative predictive value of AWE to be 94.9\%. Similar results were reported by Nagahata et $\mathrm{al}^{23}$ who found that strong or faint AWE was seen in only $4.8 \%$ and $13.3 \%$ of unruptured aneurysms, respectively. An aneurysm seen on high-resolution VWI without AWE would suggest that conservative management may be a more appropriate strategy.

In contrast, our study found a low positive predictive value of AWE and aneurysm instability (14.4\%), indicating that less than one-sixth of aneurysms that demonstrate wall enhancement may actually be unstable. These findings suggest that for unruptured, incidental aneurysms, the presence of wall enhancement is not necessarily a marker of impending aneurysm instability. Ultimately, more studies with longitudinal follow-up are needed to determine the relevance of AWE and its relationship to aneurysm instability.

Several prior reports have indicated a role for VWI in screening aneurysms. ${ }^{11,24}$ These findings must be carefully interpreted. "Instability" most commonly refers to aneurysms that have ruptured, are growing, or are symptomatic. ${ }^{9,13}$ Ruptured aneurysms likely represent a distinct underlying mechanism of enhancement; moreover, their ruptured status likely already indicates that intervention is necessary. Similarly, aneurysms with symptomatic manifestations are detected by their symptomatic nature and typically warrant treatment as well. Because ruptured or symptomatic aneurysms already indicate that an aneurysm requires close follow-up and/or treatment, inclusion of such aneurysms into a 
general group of unstable aneurysms may play a confounding role in determining the utility of VWI for detecting aneurysms at risk for growth/rupture. Grouped analyses of unstable aneurysms may, therefore, be less than ideal in determining the utility of VWI for detecting aneurysms at risk for progression/rupture. It is, thus, important that future analyses consist of explicit definitions of what is considered an unstable aneurysm to determine the utility of VWI in detecting at-risk aneurysms. Ideally, future longitudinal prospective studies with longer follow-up ( $\geq 3$ years) should be pursued to interrogate growing-versus-nongrowing aneurysms in the context of AWE to further elucidate this potential relationship.

Although our results demonstrate that AWE on initial imaging studies is not necessarily a marker of impending instability, VWI may be important to establish a baseline level of AWE. In a recent study by Gariel et al, ${ }^{25}$ increased AWE on follow-up imaging relative to baseline was predictive of aneurysm growth during a 2 -year period. Furthermore, the specificity and positive predictive value for aneurysm growth of AWE was $100 \%$ and $96 \%$, respectively. These results imply that increased AWE on followup imaging studies is a strong marker of instability and that such aneurysms likely warrant intervention. In this regard, although AWE on initial imaging studies may not be strongly predictive of future instability, obtaining VWI on initial MR imaging studies likely serves an important role in establishing a baseline degree of AWE for future comparisons. Of note, the study by Gariel et al was not included in our analysis because the included patients were a subgroup of a larger cohort studied in a separate report. ${ }^{16}$

The pathophysiologic process of aneurysm formation and progression is largely based on inflammation. Altered flow dynamics within the intracerebral vasculature results in inflammatory cell infiltration into the media and adventitia, which releases damaging proteinases and other biochemical signals that result in vessel wall weakening. ${ }^{26}$ This, in turn, promotes aneurysmal formation, growth, and rupture. Aneurysm wall enhancement is thought secondary to vessel wall inflammation and vasa vasorum proliferation, though theoretically it may also be due to endothelial disruption of the aneurysm wall resulting in contrast permeation into the vessel. ${ }^{10}$ Several studies have performed radiologicpathologic correlation analyses, which support an inflammatory hypothesis. ${ }^{27-29}$ However, the VWI protocols vary across studies, and most use low-resolution imaging. Furthermore, most studies also do not use additional flow-suppression sequences: It is wellunderstood that intra-aneurysmal slow flow adjacent to the aneurysm wall is associated with AWE, particularly in larger aneurysms in which higher degrees of flow disturbance are more likely to be encountered. ${ }^{12}$ It is therefore possible that enhancement may actually represent slow flow adjacent to the aneurysm wall and not true enhancement of the wall itself. This possibility emphasizes the importance of using imaging protocols with flow suppression in future studies. The association between AWE and wall inflammation remains incompletely characterized.

\section{Limitations}

This meta-analysis included only 3 studies. However, with limited longitudinal data, performing periodic analyses such as this is important in order interrogate the utility of high- resolution VWI for aneurysm characterization. Of the included studies, there was heterogeneity in the type and spatial resolution of imaging sequences used as well as follow-up intervals. The use of a prepulse flow-suppression modification was not specified in the Methods of the included studies. There was also heterogeneity in the definition of AWE: Some studies included only aneurysms with circumferential wall enhancement, while other studies included aneurysms with any degree of AWE. Given the lack of patient-specific data, we could not adjust the analysis for potential confounders. Only 1 study reported the size of aneurysms with enhancement versus those without. ${ }^{19}$

\section{CONCLUSIONS}

With a high negative predictive value, lack of AWE strongly indicates longitudinal aneurysm stability with the previously mentioned limitations in mind. Further prospective, longitudinal studies with strict definitions of unstable aneurysms using prepulse flow-suppression protocols are required to draw more robust conclusions.

\section{ACKNOWLEDGMENTS}

The authors would like to thank Patricia Erwin, the Mayo Clinic librarian, for performing the literature search.

Disclosures: Giuseppe Lanzino-UNRELATED: Board Membership: Superior Medical Editing, Nested Knowledge.

\section{REFERENCES}

1. Vlak MH, Algra A, Brandenburg R, et al. Prevalence of unruptured intracranial aneurysms, with emphasis on sex, age, comorbidity, country, and time period: a systematic review and meta-analysis. Lancet Neurol 2011;10:626-36 CrossRef Medline

2. Backes D, Vergouwen MD, Velthuis BK, et al. Difference in aneurysm characteristics between ruptured and unruptured aneurysms in patients with multiple intracranial aneurysms. Stroke 2014;45:12991303 CrossRef Medline

3. Ujiie $\mathrm{H}$, Tamano $\mathrm{Y}$, Sasaki $\mathrm{K}$, et al. Is the aspect ratio a reliable index for predicting the rupture of a saccular aneurysm? Neurosurgery 2001;48:495-502; discussion 502-03 CrossRef Medline

4. Greving JP, Wermer MJ, Brown RD, Jr, et al. Development of the PHASES score for prediction of risk of rupture of intracranial aneurysms: a pooled analysis of six prospective cohort studies. Lancet Neurol 2014;13:59-66 CrossRef Medline

5. Broderick JP, Viscoli CM, Brott T, et al; Hemorrhagic Stroke Project Investigators. Major risk factors for aneurysmal subarachnoid hemorrhage in the young are modifiable. Stroke 2003;34:1375-81 CrossRef Medline

6. Longstreth WT Jr, Nelson LM, Koepsell TD, et al. Cigarette smoking, alcohol use, and subarachnoid hemorrhage. Stroke 1992;23:1242-49 CrossRef Medline

7. FröSen J, Piippo A, Paetau A, et al. Remodeling of saccular cerebral artery aneurysm wall is associated with rupture: histological analysis of 24 unruptured and $\mathbf{4 2}$ ruptured cases. Stroke 2004;35:2287-93 CrossRef Medline

8. Kanematsu Y, Kanematsu M, Kurihara C, et al. Critical roles of macrophages in the formation of intracranial aneurysm. Stroke 2011;42:173-78 CrossRef

9. Hu P, Yang Q, Wang DD, et al. Wall enhancement on high-resolution magnetic resonance imaging may predict an unsteady state of 
an intracranial saccular aneurysm. Neuroradiology 2016;58:979-85 CrossRef Medline

10. Omodaka S, Endo H, Niizuma K, et al. Circumferential wall enhancement on magnetic resonance imaging is useful to identify rupture site in patients with multiple cerebral aneurysms. Neurosurgery 2018;82:638-44 CrossRef Medline

11. Texakalidis P, Hilditch CA, Lehman V, et al. Vessel wall imaging of intracranial aneurysms: systematic review and meta-analysis. World Neurosurg 2018;117:453-58 CrossRef Medline

12. Cornelissen BM, Leemans EL, Coolen BF, et al. Insufficient slowflow suppression mimicking aneurysm wall enhancement in magnetic resonance vessel wall imaging: a phantom study. Neurosurg Focus 2019;47:E19 CrossRef Medline

13. Lehman VT, Brinjikji W. Vessel wall imaging of unruptured intracranial aneurysms: ready for prime time? Not so fast! AJNR Am J Neuroradiol 2019;40:E26-29 CrossRef Medline

14. van den Berg R. Intracranial aneurysm wall enhancement: fact or fiction? Neuroradiology 2020;62:269-70 CrossRef Medline

15. Edjlali M, Gentric JC, Regent-Rodriguez C, et al. Does aneurysmal wall enhancement on vessel wall MRI help to distinguish stable from unstable intracranial aneurysms? Stroke 2014;45:3704-06 CrossRef Medline

16. Edjlali M, Guedon A, Ben Hassen W, et al. Circumferential thick enhancement at vessel wall MRI has high specificity for intracranial aneurysm instability. Radiology 2018;289:181-87 CrossRef Medline

17. Matsushige $\mathrm{T}$, Shimonaga $\mathrm{K}$, Ishii $\mathrm{D}$, et al. Vessel wall imaging of evolving unruptured intracranial aneurysms. Stroke 2019;50:189194 CrossRef Medline

18. Tian B, Toossi S, Eisenmenger L, et al. Visualizing wall enhancement over time in unruptured intracranial aneurysms using $3 \mathrm{D}$ vessel wall imaging. J Magn Reson Imaging 2019;50:193-200 CrossRef Medline

19. Vergouwen MD, Backes D, van der Schaaf IC, et al. Gadolinium enhancement of the aneurysm wall in unruptured intracranial aneurysms is associated with an increased risk of aneurysm instability: a follow-up study. AJNR Am J Neuroradiol 2019;40:1112-16 CrossRef Medline

20. Moher D, Liberati A, Tetzlaff J, et al; PRISMA Group. Preferred reporting items for systematic reviews and meta-analyses: the PRISMA statement. PLoS Med 2009;6:e1000097 CrossRef Medline

21. Sterne JA, Hernan MA, Reeves BC, et al. ROBINS-I: a tool for assessing risk of bias in non-randomised studies of interventions. BMJ 2016;355:i4919 CrossRef Medline

22. Omodaka S, Endo H, Niizuma $K$, et al. Circumferential wall enhancement in evolving intracranial aneurysms on magnetic resonance vessel wall imaging. J Neurosurg 2018 Oct 1. [Epub ahead of print] CrossRef Medline

23. Nagahata S, Nagahata M, Obara M, et al. Wall enhancement of the intracranial aneurysms revealed by magnetic resonance vessel wall imaging using three-dimensional turbo spin-echo sequence with motion-sensitized driven-equilibrium: a sign of ruptured aneurysm? Clin Neuroradiol 2016;26:277-83 CrossRef Medline

24. Wang GX, Wen L, Lei S, et al. Wall enhancement ratio and partial wall enhancement on MRI associated with the rupture of intracranial aneurysms. J Neurointerv Surg 2018;10:566-70 CrossRef Medline

25. Gariel F, Ben Hassen W, Boulouis G, et al. Increased wall enhancement during follow-up as a predictor of subsequent aneurysmal growth. Stroke 2020;51:1868-72 CrossRef Medline

26. Zhang X, Ares WJ, Taussky P, et al. Role of matrix metalloproteinases in the pathogenesis of intracranial aneurysms. Neurosurg Focus 2019;47:E4 CrossRef Medline

27. Hudson JS, Zanaty M, Nakagawa $D$, et al. Magnetic resonance vessel wall imaging in human intracranial aneurysms. Stroke 2018 Dec 7. [Epub ahead of print] CrossRef Medline

28. Quan K, Song J, Yang Z, et al. Validation of wall enhancement as a new imaging biomarker of unruptured cerebral aneurysm. Stroke 2019;50:1570-73 CrossRef Medline

29. Shimonaga K, Matsushige T, Ishii D, et al. Clinicopathological insights from vessel wall imaging of unruptured intracranial aneurysms. Stroke 2018;49:2516-19 CrossRef Medline 